\title{
Universe: Not expanding
}

\author{
Bandula Dahanayake \\ Farmfield Crescent, Kanata, Ontario, Canada K2M 2S9
}

Email address:

Bandula_Dahanayake@yahoo.com

To cite this article:

Bandula Dahanayake. Universe: Not Expanding. International Journal of Astrophysics and Space Science.

Vol. 2, No. 4, 2014, pp. 66-70. doi: 10.11648/j.ijass.20140204.12

\begin{abstract}
Contrary to the widespread belief, the Universe is neither expanding nor contracting. What is taking place is the orbit dilation of the galaxies. The orbit dilation is a phenomenon that results from the mass creation due to energy to mass conversion occurring in galaxies. The absence of Universe expansion indicates that the origin of the Universe cannot be traced back in time to a hypothetical Big-Bang. The Orbit Dilation Theorem and the Orbit Acceleration Theorem that describe the mechanics of orbit dilation are derived under the axiom that the mass of a galaxy or a planet is time varying and never remains a constant. These theorems are then used to demonstrate how and why galaxies are moving away always at an accelerated rate. There is absolutely no space expansion hypothesis required for determining why the distance galaxies are moving away at an accelerated rate. The galactic and planetary orbits are dynamic and are always either dilating or contracting in accordance with the rate of change of the logarithmic mass, while orbit acceleration takes place in proportion to the second derivative of the mass with respect to time per unit mass; the galactic and planetary orbits never remain static. It is also shown that as a result of orbit dilation and orbit contraction, galaxies may occasionally come into collision course with each other; however, a singular cohesive collision of the galaxies leading to a Big-Bang is improbable because it is highly unlikely that the assumption required would be supported in the nature. Hubble's velocity-distance relationship is obtained as a possible, but quite improbable special case of the Orbit Dilation Theorem; it is improbable because the assumption, the rate of change of logarithmic mass is a constant, that had to be made cannot be expected to satisfy in nature. The acceleration of a galaxy in Hubble relationship is the square of the Hubble Constant times the distance to the galaxy. The Orbit Dilation Theorem and the Orbit Acceleration Theorem are also used to analyze the behavior of the solar system. The orbit contraction is prevalent in planetary systems as a result of mass loss due to mass to energy conversion, especially due to radiation and non-renewable resource depletion. The earth's orbit contraction leads to global warming, as well as, to the gradual shortening of the year. It is possible to counter balance the orbit contraction and resulting global warming by limiting the depletion of non-renewable resources, as well as by protecting and sustaining the mass creation or the energy to mass conversion that is naturally taking place in the bio-mass.
\end{abstract}

Keywords: Non-Expanding-Universe, Hubble-Constant, Global-Warming, Big-Bang, Red-Shift

\section{Introduction}

As early as 1920 s, using the red shift of the light spectrum received from distant galaxies, it was observed that the distant galaxies were moving away $[3,1]$. Though it is very difficult to fathom, this observation had been used in the past to postulate that the Universe is expanding $[5,3]$. Later, it was observed that the red shift of the light spectrum received from distant galaxies is increasing [4]. The increasing red shift of the light spectrum of the distant galaxies was used to hypothesize that the Universe is expanding at an accelerated rate $[5,4]$. The idea of expanding Universe also led to the conceptualization that the Universe was originated from a singular explosion came to known as Big-Bang [3, 2, 4, 7].

In this paper, the validity of expanding Universe, and the Big-Bang were questioned, and re-examined. The axiom of ever changing mass (mass-energy duality) of the cosmic bodies is used to derive the necessary relationships to explain why distant galaxies are moving away at an accelerated rate; this derivation does not require or assume any space expansion hypothesis. The same axiom of continuous time varying nature of the masses of the planets is used to investigate the cause of global warming and ways to combat it; it is also used to explain the gradual shortening of the year. 


\section{Model}

Let $\mathrm{O}$ be the orbiting center of the galaxies. All the orbital distances are measured with respect to $\mathrm{O}$.

Consider a galaxy $\mathrm{G}_{\mathrm{i}}\left[\mathrm{r}_{\mathrm{i}}(\mathrm{t}), \mathrm{v}_{\mathrm{i}}(\mathrm{t}), \mathrm{M}_{\mathrm{i}}(\mathrm{t}), \mathrm{u}_{\mathrm{i}}(\mathrm{t})\right], \forall \mathrm{i}=1,2,3, \ldots$ at time $\mathrm{t}$, where,

$\mathrm{r}_{\mathrm{i}}(\mathrm{t})=$ orbit distance, i.e. the distance to the galaxy from $\mathrm{O}$, $\mathrm{v}_{\mathrm{i}}(\mathrm{t})=$ orbital speed, i.e. the speed at which the galaxy is orbiting around $\mathrm{O}$,

$\mathrm{M}_{\mathrm{i}}(\mathrm{t})=$ mass of the galaxy,

$\mathrm{u}_{\mathrm{i}}(\mathrm{t})=\frac{d}{d t} \mathrm{r}_{\mathrm{i}}(\mathrm{t})$, i.e. the speed of orbit dilation.

Orbit acceleration is given by, $\frac{d}{d t} \mathrm{u}_{\mathrm{i}}(\mathrm{t})$.

Axiom

The mass of a galaxy or a planet is time varying, and never a constant in time.

Orbit Dilation Theorem

The speed of orbit dilation, $\mathrm{u}_{\mathrm{i}}(\mathrm{t})$ of the galaxy $\mathrm{G}_{\mathrm{i}}$ is given by,

$$
\mathrm{u}_{\mathrm{i}}(\mathrm{t})=\mathrm{H}_{\mathrm{i}}(\mathrm{t}) \mathrm{r}_{\mathrm{i}}(\mathrm{t})
$$

where, the Orbit Dilation Parameter $\mathrm{H}_{\mathrm{i}}(\mathrm{t})$ is,

$$
\mathrm{H}_{\mathrm{i}}(\mathrm{t})=2 \frac{d}{d t} \ln \mathrm{M}_{\mathrm{i}}(\mathrm{t}), \quad \forall \mathrm{i}=1,2,3, \ldots .
$$

The proof is given in the Appendix-A.

Property-1

Farther the galaxy away, faster is the orbit dilation and hence faster it is moving away.

Corollary-1

$\mathrm{H}_{\mathrm{i}}(\mathrm{t})$ is a constant only when the rate of change of logarithmic mass is a constant.

Corollary-2

Planetary and galactic orbits are always either dilating or contracting, but never remains static.

Orbit Acceleration Theorem

Orbit acceleration $\frac{d}{d t} \mathrm{u}_{\mathrm{i}}(\mathrm{t})$ of galaxy $\mathrm{G}_{\mathrm{i}}$ is given by,

$$
\frac{d}{d t} \mathrm{u}_{\mathrm{i}}(\mathrm{t})=\gamma_{\mathrm{i}}(\mathrm{t}) \mathrm{r}_{\mathrm{i}}(\mathrm{t})
$$

where, the Orbit Acceleration Parameter $\gamma_{i}(t)$ is a quadratic function of Orbit Dilation Parameter $\mathrm{H}_{\mathrm{i}}(\mathrm{t})$,

$$
\begin{gathered}
\gamma_{\mathrm{i}}(\mathrm{t})=\mathrm{H}_{\mathrm{i}}^{2}(\mathrm{t})+\frac{d}{d t} \mathrm{H}_{\mathrm{i}}(\mathrm{t}), \\
\gamma_{\mathrm{i}}(\mathrm{t}) \cong\left[2 / \mathrm{M}_{\mathrm{i}}(\mathrm{t})\right] \frac{d}{d t}\left[\frac{d}{d t} \mathrm{M}_{\mathrm{i}}(\mathrm{t})\right] .
\end{gathered}
$$

The proof is given in Appendix-B.

\section{Property-2}

Farther the Galaxy away, faster is its acceleration.

\section{Corollary-3}

Since $\gamma_{\mathrm{i}}(\mathrm{t})=\mathrm{H}_{\mathrm{i}}^{2}(\mathrm{t})+\frac{d}{d t} \mathrm{H}_{\mathrm{i}}(\mathrm{t})$, for any given $\mathrm{H}_{\mathrm{i}}(\mathrm{t}) \neq 0, \exists \mathrm{a}$ $\gamma_{i}(t) \neq 0$; hence, the speed of orbit dilation is never a constant; orbits always dilate at an accelerated rate; the galaxies always move away at an accelerated speed.

These corollaries follow directly from the Orbit Dilation Theorem and the Orbit Acceleration Theorem.
Now, we can analyze the dynamics of galaxies, as well as our solar system using the Orbit Dilation Parameter $\mathrm{H}_{\mathrm{i}}(\mathrm{t})$, and the Orbit Acceleration Parameter $\gamma_{i}(t)$,

$$
\begin{gathered}
\mathrm{H}_{\mathrm{i}}(\mathrm{t})=\left[2 / \mathrm{M}_{\mathrm{i}}(\mathrm{t})\right] \frac{d}{d t} \mathrm{M}_{\mathrm{i}}(\mathrm{t}), \\
\gamma_{\mathrm{i}}(\mathrm{t}) \cong\left[2 / \mathrm{M}_{\mathrm{i}}(\mathrm{t})\right] \frac{d}{d t}\left[\frac{d}{d t} \mathrm{M}_{\mathrm{i}}(\mathrm{t})\right],
\end{gathered}
$$

Exact relationship for $\gamma_{\mathrm{i}}(\mathrm{t})$ is given by (Appendix-B),

$$
\gamma_{\mathrm{i}}(\mathrm{t})=\left[2 / \mathrm{M}_{\mathrm{i}}(\mathrm{t})\right] \frac{d}{d t}\left[\frac{d}{d t} \mathrm{M}_{\mathrm{i}}(\mathrm{t})\right]+2\left[\frac{d}{d t} \ln \mathrm{M}_{\mathrm{i}}(\mathrm{t})\right]^{2}
$$

\section{Analysis}

Though $\mathrm{H}_{\mathrm{i}}(\mathrm{t})$ resembles the Hubble Constant $[1,2,8]$, the $\mathrm{H}_{\mathrm{i}}(\mathrm{t})$ here is not a constant. The $\mathrm{H}_{\mathrm{i}}(\mathrm{t})$ is proportional to the rate of change of logarithmic mass of the galaxy $\mathrm{G}_{\mathrm{i}}(\mathrm{t})$, at time $\mathrm{t}$.

Case-1: $\frac{d}{d t} \mathrm{M}_{\mathrm{i}}(\mathrm{t})>0$.

In this case, $H_{i}(t)>0$. Mass creation due to mass energy conversion is taking place in this galaxy, and hence its orbit dilates at speed $u_{i}(t)>0$. The galactic orbit will dilate at an accelerated rate given by the Orbit Acceleration Theorem. As a result, the galaxy will be moving away from $\mathrm{O}$ at an accelerated rate. As the mass increases, due to the energy to mass conversion, the galaxy gradually cools down while moving away from $\mathrm{O}$ until $\frac{d}{d t} \mathrm{M}_{\mathrm{i}}(\mathrm{t})=0$.

Case-2: $\frac{d}{d t} \mathrm{M}_{\mathrm{i}}(\mathrm{t})=0$.

In this case, $\mathrm{H}_{\mathrm{i}}(\mathrm{t})=0$, and $\mathrm{u}_{\mathrm{i}}(\mathrm{t})=0$. This galaxy is in a transient state at this time, because mass never remains a constant. At this point onward, the mass to energy conversion due to radiation prevails, and mass decreases, hence,

$$
\frac{d}{d t} \mathrm{M}_{\mathrm{i}}(\mathrm{t})<0
$$

Case- $3: \frac{d}{d t} \mathrm{M}_{\mathrm{i}}(\mathrm{t})<0$.

In this case, $\mathrm{H}_{\mathrm{i}}(\mathrm{t})<0$, and hence $\mathrm{u}_{\mathrm{i}}(\mathrm{t})<0$. This galaxy is losing mass due to mass to energy conversion, especially due to the radiation loss. This in turn leads to the galactic orbit contraction. As a result, the galaxy will be moving back toward $\mathrm{O}$. The inward moving galaxy may ultimately collide with an outward moving galaxy creating a new hot galaxy with new planetary systems.

Case-4: $\mathrm{H}_{\mathrm{i}}(\mathrm{t})=\mathrm{H}_{\mathrm{j}}(\mathrm{t}), \mathrm{r}_{\mathrm{i}}(\mathrm{t})>\mathrm{r}_{\mathrm{j}}(\mathrm{t})$.

In this case, $u_{i}(t) / u_{j}(t)=r_{i}(t) / r_{j}(t)>1$. That is, farther the galaxy away from $\mathrm{O}$ faster is the orbital dilation, and hence faster it is moving away from $\mathrm{O}$. The same hold true for orbit contraction.

Case- $5: \mathrm{r}_{\mathrm{i}}(\mathrm{t})=\mathrm{r}(\mathrm{t}), \forall \mathrm{i}=1,2,3, \ldots$.

In this case, all the galaxies are on a surface of a sphere of radius $\mathrm{r}(\mathrm{t})$. They all have distinct orbits, and orbit distances from $\mathrm{O}$ are the same $\mathrm{r}(\mathrm{t})$. Since $\mathrm{H}_{\mathrm{i}}(\mathrm{t})>0$, is different $\forall \mathrm{i}=1,2,3, \ldots$, the orbits dilate at different speeds, and soon the orbit distances will be different leading to $\mathrm{r}_{\mathrm{i}} \neq \mathrm{r}_{\mathrm{j}}, \forall \mathrm{i}, \mathrm{j}=1$, $2,3, \ldots$. Let $\mathrm{r}_{\text {ave }}(\mathrm{t})=\operatorname{average}\left[\mathrm{r}_{1}(\mathrm{t}), \mathrm{r}_{2}(\mathrm{t}), \ldots.\right]$, then, we know 
that as $r_{\text {ave }}(t)$ increases, surface area of the sphere with radius $r_{\text {ave }}(t)$ increases. If we assume that all the galaxies are approximately on the surface of the sphere with radius $r_{a v e}(t)$, i.e. the galaxies are more or less on the surface of an expanding balloon of radius $\mathrm{r}_{\mathrm{ave}}(\mathrm{t})$, then, any observer in any one of the galaxies sees that other galaxies are speeding away at an accelerated rate. Farther a galaxy away from the observer, the faster it is moving away from the observer.

Case- $6: \mathrm{r}_{\mathrm{i}}(\mathrm{t})=\mathrm{r}(\mathrm{t}), \mathrm{H}_{\mathrm{i}}(\mathrm{t})=\mathrm{H}(\mathrm{t}), \forall \mathrm{i}=1,2,3, \ldots$.

This is a possible but very improbable case, where all the galaxies are orbiting around $\mathrm{O}$ on a sphere. They all have distinct orbits, with the same orbit distance $\mathrm{r}(\mathrm{t})$ from $\mathrm{O}$. Since, $r_{i}(t)=r(t)$, and $H_{i}(t)=H(t)>0, \forall i=1,2,3, \ldots$, all the galaxies dilate at the same speed with the same acceleration, or the synchronized dilation takes place. As the orbits dilate, the orbit distances of all the galaxies remain the same, $r(t)$. This is analogous to the situation where all the galaxies are on a surface of an expanding balloon of radius $r(t)$. The galaxies move away from each other as orbits dilate (or the balloon expands), and all the galaxies will remain on the expanding sphere at any time t. An observer in any one of the galaxies sees that the galaxies are moving away from the observer at an accelerated rate. In addition, farther a galaxy away from the observer, faster it is moving away.

With all the galaxies on the surface, the balloon keeps expanding until $\mathrm{H}(\mathrm{t})=0$, or nor more mass creation due to energy to mass conversion takes place, at which point shrinking of the balloon takes place, since $\mathrm{H}(\mathrm{t})<0$ as mass loss takes place due to mass to energy conversion, especially when mass loss due to radiation becomes dominant. All the orbits of the galaxies are now contracting at the same speed while maintaining same orbital distance, $\mathrm{r}(\mathrm{t})$; in other words, synchronous contraction is taking place (i.e. the balloon having galaxies on its surface is now shrinking). As $r(t)$ shrinks, all the galaxies will ultimately collide together leading to a Big-Bang. This singular collision happens, not because of any space expansion or contraction. It happens solely due to the orbit dilation followed by orbit contraction under very special circumstance, where they all have same orbit distance and the same orbit dilation parameter $\mathrm{H}(\mathrm{t})$.

However, conditions $\mathrm{r}_{\mathrm{i}}(\mathrm{t})=\mathrm{r}(\mathrm{t}), \mathrm{H}_{\mathrm{i}}(\mathrm{t})=\mathrm{H}(\mathrm{t}), \forall \mathrm{i}=1,2,3, \ldots$ are possible but highly theoretical and improbable. To achieve these conditions, not only all the galaxies must be on a sphere of radius $r(t)$, they must also have the same rate of change of the logarithmic mass, which is quite improbable, and difficult to satisfy. Therefore, such a singular collision of the galaxies leading to a Big-Bang is not plausible.

\section{Observations}

Now, let us consider a hypothetical situation where we place an observer at $\mathrm{O}$. What does the observer see? The observer will see an increasing red shift in the light spectrum of the galaxies. In other words, observer sees that the galaxies are moving away at an accelerated speed. As the Orbit Dilation Theorem demonstrated, the galaxies are moving away from the observer because of the Orbit

\section{Dilation.}

Now, consider the observers on earth in our Milky-way galaxy. Time and time again, observers on earth have found that the galaxies are moving away at an accelerated rate. The first to observe that were Vesto Slipher, Carl Wilhelm Wirtz, and Edward Hubble [3, 5].

Observers on earth see the exactly the same phenomenon that the hypothetical observers at $\mathrm{O}$ see. That means, the Milky-way galaxy must be close to $\mathrm{O}$. So, we can assume that,

$\mathrm{r}_{\mathrm{i}}(\mathrm{t}) \approx$ distance from earth to the Galaxy $\mathrm{G}_{\mathrm{i}}$

$\mathrm{u}_{\mathrm{i}}(\mathrm{t}) \approx$ speed the galaxy $\mathrm{G}_{\mathrm{i}}(\mathrm{t})$ moving away from us.

Now, if $\mathrm{H}_{\mathrm{i}}(\mathrm{t})=2 \frac{d}{d t} \ln \mathrm{M}_{\mathrm{i}}(\mathrm{t})=\mathrm{H}$, a constant, i.e. the rate of change of logarithmic mass is a constant $H$, then, the Orbit Dilation Theorem will be the same as the Hubble's velocity-distance relationship $[1,2]$ where $\mathrm{H}$ being the Hubble Constant.

Using the Orbit Acceleration Theorem, we can obtain the Galactic Acceleration Parameter $\gamma_{i}(t)$ when the Hubble's velocity-distance relationship holds, i.e. when $\mathrm{H}_{\mathrm{i}}(\mathrm{t})=\mathrm{H}$, the Hubble Constant. From Orbit Acceleration Theorem, we know that,

$$
\begin{gathered}
\gamma_{\mathrm{i}}(\mathrm{t})=\mathrm{H}_{\mathrm{i}}{ }^{2}(\mathrm{t})+\frac{d}{d t} \mathrm{H}_{\mathrm{i}}(\mathrm{t}) . \\
\text { If } \mathrm{H}_{\mathrm{i}}(\mathrm{t})=\mathrm{H} \text {, then, } \frac{d}{d t} \mathrm{H}_{\mathrm{i}}(\mathrm{t})=0 \text {, and hence we get, } \\
\gamma_{\mathrm{i}}(\mathrm{t})=\mathrm{H}^{2}=[\text { Hubble Constant }]^{2},
\end{gathered}
$$

i.e the Galactic Acceleration Parameter is equal to the square of the Hubble Constant.

If Hubble's velocity-distance relationship holds, then the acceleration of a galaxy will be given by $\mathrm{H}^{2}$ times the distance to the galaxy, i.e.,

$$
\begin{aligned}
\frac{d}{d t} \mathrm{u}_{\mathrm{i}}(\mathrm{t}) & =\mathrm{H}^{2} \mathrm{r}_{\mathrm{i}}(\mathrm{t}), \\
\mathrm{u}_{\mathrm{i}}(\mathrm{t}) & =\mathrm{Hr}_{\mathrm{i}}(\mathrm{t}),
\end{aligned}
$$

where, $\mathrm{H}^{2}$ is the Galactic Acceleration Parameter.

However, $\mathrm{H}_{\mathrm{i}}(\mathrm{t})$ is not a constant in general, because there is no reason for the rate of change of logarithmic mass to be a constant in nature.

\section{Our Solar System}

The Orbit Dilation Theorem is not limited to galaxies. It equally applies to any planetary system, including our solar system.

The Orbit Dilation Theorem for the solar system is,

$$
\begin{aligned}
\mathrm{u}_{\mathrm{i}}(\mathrm{t}) & =\mathrm{H}_{\mathrm{i}}(\mathrm{t}) \mathrm{r}_{\mathrm{i}}(\mathrm{t}), \\
\mathrm{H}_{\mathrm{i}}(\mathrm{t}) & =2 \frac{d}{d t} \ln \mathrm{M}_{\mathrm{i}}(\mathrm{t}) ;
\end{aligned}
$$

the Orbit Acceleration Theorem for the solar system is,

$$
\frac{d}{d t} \mathrm{u}_{\mathrm{i}}(\mathrm{t})=\gamma_{\mathrm{i}}(\mathrm{t}) \mathrm{r}_{\mathrm{i}}(\mathrm{t})
$$




$$
\gamma_{\mathrm{i}}(\mathrm{t}) \cong\left[2 / \mathrm{M}_{\mathrm{i}}(\mathrm{t})\right] \frac{d}{d t}\left[\frac{d}{d t} \mathrm{M}_{\mathrm{i}}(\mathrm{t})\right], \quad \forall \mathrm{i}=1,2,3, \ldots,
$$

where, $M_{i}(t)$ is the mass of the $i^{\text {th }}$ planet,

$\mathrm{u}_{\mathrm{i}}(\mathrm{t})$ is the speed of orbit dilation/contraction, $r_{i}(t)$ is the orbital distance of the $i^{\text {th }}$ planet from the sun, $\frac{d}{d t} \mathrm{u}_{\mathrm{i}}(\mathrm{t})$ is the orbit acceleration/deceleration, at time $\mathrm{t}$.

In the case of planets, what is prevalent is mass loss due to mass to energy conversion, i.e.

$\frac{d}{d t} \mathrm{M}_{\mathrm{i}}(\mathrm{t})<0$, making $\mathrm{H}_{\mathrm{i}}(\mathrm{t})<0$, causing orbits to contract.

This orbit contraction moves the planets toward the sun. Both the speed of orbit dilation, as well as the orbit acceleration of a planet are proportional to the orbit distance of the planet, $r_{i}(t)$. Farther a planet away, faster it is moving toward the sun; on the other hand, closer a planet to the sun, slower it is moving toward the sun. This assures the longevity of the planets in orbits without being swallowed up by the sun and becoming fuel for the sun too fast. However, ultimately, every planet will end up in the sun, due to $\mathrm{H}_{\mathrm{i}}(\mathrm{t})<0$, and its resulting continuous orbit contraction. That is the unavoidable destiny of all the planets; how fast that happens is determined by the magnitude of $\mathrm{H}_{\mathrm{i}}(\mathrm{t})$.

Property-3

Orbit contraction leads to global warming.

Property-4

Orbit contraction shortens the year.

As with any other planet, the earth also undergoes the same orbit contraction as a result of mass loss due to mass energy conversion. The mass loss is most dominant at the core of the earth, mainly due to atomic radiation that keeps the core melting [6]. The mass loss due to radiation is also prevalent in the rocks and on the surface of the earth too. That is a natural phenomenon which we have no control, that drives $\mathrm{H}_{\mathrm{i}}(\mathrm{t})$ negative, giving rise to orbit contraction and resulting global warming. However, we have the ability to counter balance the orbit contraction with orbit dilation. The less we consume non-renewable resources, less negative $\mathrm{H}_{\mathrm{i}}(\mathrm{t})$ becomes. The bio-mass, on the other hand, have the ability convert the sun's energy into mass, driving $\mathrm{H}_{\mathrm{i}}(\mathrm{t})$ in the positive direction. So, by enhancing the mass creation from energy to mass conversion (i.e. by enhancing and sustaining the bio-mass, and reducing its destruction), and limiting the non-renewable mass depletion, we can reduce the negative shift of $\mathrm{H}_{\mathrm{i}}(\mathrm{t})$, in effect, curtailing the speed of global warming.

Further, as mass decreases, in addition to orbit contraction, the orbiting speed increases. These in turn reduce the time it takes for earth to complete a single orbit leading to gradual shortening of the year, probably a few seconds a year. Though not so significant during the human life span, the more negative the $\mathrm{H}_{\mathrm{i}}(\mathrm{t})$ is, the faster we age. The cumulative effect of shortening of the year will be significant as time passes in millions or billions of years. This can be overcome by pushing $\mathrm{H}_{\mathrm{i}}(\mathrm{t})$ toward the positive direction; we have limited ability to do that by conserving the bio-mass and reducing the depletion of hydrocarbon resources.

On a side note, the real reason for the extinction of dinosaurs would have been their inability adapt in time with the environmental changes brought by millions of years of cumulative effect of earth's orbit contraction. Perhaps, their massive size might have played a role in their inept ability to adapt. Though those environments were detrimental to dinosaurs, they must have been quite favorable for new life forms that evolved into human species to emerge.

The devastating effect of accumulated orbit contraction is also very evident from the disappearance of glaciers from the ice age. Once, most of the northern hemisphere was covered in ice. Melting of these glaciers can be attributed to the global warming that resulted from orbit contraction.

Since we have no control of atomic radiation loss, as mass is converted to energy, the unstoppable orbit contraction will gradually move the earth away from the life sustainable Goldilocks zone toward the sun, making the life on earth unsustainable. This is further accelerated due to our inability to limit the consumption of non-renewable natural resources, as well as our failure to protect the bio-mass. As the earth makes its way toward the sun, it will gradually become a red hot planet. However, the same orbital contraction may bring a smaller distant planet into Goldilocks zone providing suitable natural environment for new life forms to evolve. Similarly, the orbit contraction will gradually bring Mercury closer and closer to the sun; in the end, Mercury will be swallowed by the sun. Ultimately, it is not just Mercury, one by one, all the planets will be fuelling the sun due to orbit contraction in cosmological time; how fast or how slow that happens to a particular planet is determined by its Orbit Dilation Parameter $\mathrm{H}_{\mathrm{i}}(\mathrm{t})$ and the Orbit Acceleration Parameter $\gamma_{\mathrm{i}}(\mathrm{t})$.

\section{Conclusions}

Space is neither expanding nor contracting. Universe is neither expanding nor contracting. Orbits of galaxies and planets are either dilating or contracting. In the absence of an expanding Universe, the origin of the Universe cannot be traced back in time to a hypothetical Big-Bang; in light of this, Big-Bang is not a plausible phenomenon. However, some minor-Bangs of sort are possible from galactic collisions. A cohesive singular collision of galaxies leading to a Big-Bang due to orbit contraction is theoretically possible, but practically highly improbable.

The Orbit Dilation Theorem and the Orbit Acceleration Theorem provide galactic and planetary dynamics in describing the effect of the rate of change of mass on galactic and planetary orbits. The orbit dilation moves a galaxy away from orbital center always at an accelerated rate. The orbit contraction brings a galaxy toward the orbital center.

Due to the energy to mass conversion or mass creation taking place in the galaxies, their orbits dilate, and hence the galaxies are moving away at an accelerated rate. There is no space expansion of any sort.

Earth and the rest of the planets are moving toward the sun due to orbit contraction. The earth's orbit contraction causes the global warming, and shortening of the year. By enhancing the energy to mass conversion (i.e. by protecting 
the bio-mass, the forest), and decreasing the depletion of non-renewable resources, we can minimize the orbit contractions, and in effect reduce the global warming and shortening of the year.

\section{Appendix-A}

\section{The Proof of Orbit Dilation Theorem}

The galaxy $\mathrm{G}_{\mathrm{i}}$ at time $\mathrm{t}$ is defined by, $\mathrm{G}_{\mathrm{i}}\left[\mathrm{r}_{\mathrm{i}}(\mathrm{t}), \mathrm{v}_{\mathrm{i}}(\mathrm{t}), \mathrm{M}_{\mathrm{i}}(\mathrm{t})\right]$, $\forall \mathrm{i}=1,2,3, \ldots$.

The galaxy $\mathrm{G}_{\mathrm{i}}$ at time $\mathrm{t}+\Delta \mathrm{t}$, is given by,

$\mathrm{G}_{\mathrm{i}}\left[\mathrm{r}_{\mathrm{i}}(\mathrm{t}+\Delta \mathrm{t}), \mathrm{v}_{\mathrm{i}}(\mathrm{t}+\Delta \mathrm{t}), \mathrm{M}_{\mathrm{i}}(\mathrm{t}+\Delta \mathrm{t})\right], \forall \mathrm{i}=1,2,3, \ldots$, , where,

$$
r_{i}(t+\Delta t)=r_{i}(t)+\Delta r_{i}(t)
$$

$\mathrm{v}_{\mathrm{i}}(\mathrm{t}+\Delta \mathrm{t})=\mathrm{v}_{\mathrm{i}}(\mathrm{t})+\Delta \mathrm{v}_{\mathrm{i}}(\mathrm{t})$

$\mathrm{M}_{\mathrm{i}}(\mathrm{t}+\Delta \mathrm{t})=\mathrm{M}_{\mathrm{i}}(\mathrm{t})+\Delta \mathrm{M}_{\mathrm{i}}(\mathrm{t})$, and $\Delta \mathrm{t}$ is an arbitrarily small time interval.

The conservation of momentum at time $t$ and $t+\Delta t$ gives,

$$
M_{i}(t+\Delta t) v_{i}(t+\Delta t)=M_{i}(t) v_{i}(t)
$$

Using the gravitational laws, one can obtain the relationship,

$$
r_{i}(t+\Delta t) / r_{i}(t)=\left[v_{i}(t) / v_{i}(t+\Delta t]^{2}\right.
$$

Combining eqns. (A1) and (A2), we get,

$$
\begin{aligned}
r_{i}(t+\Delta t) & =\left[M_{i}(t+\Delta t) / M_{i}(t)\right]^{2} r_{i}(t) \\
& =\left[1+\Delta M_{i}(t) / M_{i}(t)\right]^{2} r_{i}(t)
\end{aligned}
$$

Since $\Delta \mathrm{M}_{\mathrm{i}}(\mathrm{t}) \ll \mathrm{M}_{\mathrm{i}}(\mathrm{t})$, and $\mathrm{r}_{\mathrm{i}}(\mathrm{t}+\Delta \mathrm{t})=\mathrm{r}_{\mathrm{i}}(\mathrm{t})+\Delta \mathrm{r}_{\mathrm{i}}(\mathrm{t})$, eqn. (A3) becomes,

$$
\mathrm{r}_{\mathrm{i}}(\mathrm{t})+\Delta \mathrm{r}_{\mathrm{i}}(\mathrm{t})=\left[1+2 \Delta \mathrm{M}_{\mathrm{i}}(\mathrm{t}) / \mathrm{M}_{\mathrm{i}}(\mathrm{t})\right] \mathrm{r}_{\mathrm{i}}(\mathrm{t})
$$

i.e., $\Delta \mathrm{r}_{\mathrm{i}}(\mathrm{t})=\left[2 \Delta \mathrm{M}_{\mathrm{i}}(\mathrm{t}) / \mathrm{M}_{\mathrm{i}}(\mathrm{t})\right] \mathrm{r}_{\mathrm{i}}(\mathrm{t})$

Dividing by $\Delta \mathrm{t}$, we get,

$$
\Delta \mathrm{r}_{\mathrm{i}}(\mathrm{t}) / \Delta \mathrm{t}=\left[\left(2 / \mathrm{M}_{\mathrm{i}}(\mathrm{t})\right)\left(\Delta \mathrm{M}_{\mathrm{i}}(\mathrm{t}) / \Delta \mathrm{t}\right)\right] \mathrm{r}_{\mathrm{i}}(\mathrm{t})
$$

By taking limits $\Delta \mathrm{t} \rightarrow 0$, we get,

$\lim _{\Delta \mathrm{t} \rightarrow 0} \Delta \mathrm{r}_{\mathrm{i}}(\mathrm{t}) / \Delta \mathrm{t}=\frac{d}{d t} \mathrm{r}_{\mathrm{i}}(\mathrm{t})$

$\lim _{\Delta \mathrm{t} \rightarrow 0} \Delta \mathrm{M}_{\mathrm{i}}(\mathrm{t}) / \Delta \mathrm{t}=\frac{d}{d t} \mathrm{M}_{\mathrm{i}}(\mathrm{t})$, i.e. the rate of change of mass of the galaxy $\mathrm{G}_{\mathrm{i}}$ at time $\mathrm{t}$.

Let, $\mathrm{u}_{\mathrm{i}}(\mathrm{t})=\frac{d}{d t} \mathrm{r}_{\mathrm{i}}$, i.e. the orbital dilation of galaxy $\mathrm{G}_{\mathrm{i}}$ at time $\mathrm{t}$.

Now, eqn. (A5) becomes,

$$
\mathrm{u}_{\mathrm{i}}(\mathrm{t})=\left[2 / \mathrm{M}_{\mathrm{i}}(\mathrm{t})\right]\left[\frac{d}{d t} \mathrm{M}_{\mathrm{i}}(\mathrm{t})\right] \mathrm{r}_{\mathrm{i}}(\mathrm{t})
$$

It is same as, $\mathrm{u}_{\mathrm{i}}(\mathrm{t})=\left[2 \frac{d}{d t} \ln \mathrm{M}_{\mathrm{i}}(\mathrm{t})\right] \mathrm{r}_{\mathrm{i}}(\mathrm{t})$

$$
\text { i.e. } u_{i}(t)=H_{i}(t) r_{i}(t)
$$

where, the Orbit Dilation Parameter $\mathrm{H}_{\mathrm{i}}(\mathrm{t})$ is given by,

$$
\mathrm{H}_{\mathrm{i}}(\mathrm{t})=2 \frac{d}{d t} \ln \mathrm{M}_{\mathrm{i}}(\mathrm{t})
$$

\section{Appendix-B}

\section{The Proof of Orbit Acceleration Theorem}

Consider the orbit dilation relationship,

$$
\mathrm{u}_{\mathrm{i}}(\mathrm{t})=\mathrm{H}_{\mathrm{i}}(\mathrm{t}) \mathrm{r}_{\mathrm{i}}(\mathrm{t})
$$

By differentiating w. r. t. time, we get,

$$
\frac{d}{d t} \mathrm{u}_{\mathrm{i}}(\mathrm{t})=\mathrm{H}_{\mathrm{i}}(\mathrm{t}) \mathrm{u}_{\mathrm{i}}(\mathrm{t})+\mathrm{r}_{\mathrm{i}}(\mathrm{t}) \frac{d}{d t} \mathrm{H}_{\mathrm{i}}(\mathrm{t})
$$

Combining eqns. (B1) and (B2), we get,

$$
\frac{d}{d t} \mathrm{u}_{\mathrm{i}}(\mathrm{t})=\gamma_{\mathrm{i}}(\mathrm{t}) \mathrm{r}_{\mathrm{i}}(\mathrm{t})
$$

where, the Orbit Acceleration Parameter, $\gamma_{i}(t)$ is,

$$
\gamma_{\mathrm{i}}(\mathrm{t})=\mathrm{H}_{\mathrm{i}}^{2}(\mathrm{t})+\frac{d}{d t} \mathrm{H}_{\mathrm{i}}(\mathrm{t})
$$

Substituting for $\mathrm{H}_{\mathrm{i}}(\mathrm{t})$, we get,

$$
\begin{gathered}
\mathrm{H}_{\mathrm{i}}^{2}(\mathrm{t})=4\left[\frac{d}{d t} \ln \mathrm{M}_{\mathrm{i}}(\mathrm{t})\right]^{2}, \\
\frac{d}{d t} \mathrm{H}_{\mathrm{i}}(\mathrm{t})=\frac{d}{d t}\left[2 \frac{d}{d t} \ln \mathrm{M}_{\mathrm{i}}(\mathrm{t})\right] \\
=\left[2 / \mathrm{M}_{\mathrm{i}}(\mathrm{t})\right] \frac{d}{d t}\left[\frac{d}{d t} \mathrm{M}_{\mathrm{i}}(\mathrm{t})\right]-2\left[\frac{d}{d t} \ln \mathrm{M}_{\mathrm{i}}(\mathrm{t})\right]^{2} .
\end{gathered}
$$

Substituting in eqn. (B3), we get,

$$
\gamma_{\mathrm{i}}(\mathrm{t})=\left[2 / \mathrm{M}_{\mathrm{i}}(\mathrm{t})\right] \frac{d}{d t}\left[\frac{d}{d t} \mathrm{M}_{\mathrm{i}}(\mathrm{t})\right]+2\left[\frac{d}{d t} \ln \mathrm{M}_{\mathrm{i}}(\mathrm{t})\right]^{2}
$$

Since, $2\left[\frac{d}{d t} \ln \mathrm{M}_{\mathrm{i}}(\mathrm{t})\right]^{2} \ll\left[2 / \mathrm{M}_{\mathrm{i}}(\mathrm{t})\right] \frac{d}{d t}\left[\frac{d}{d t} \mathrm{M}_{\mathrm{i}}(\mathrm{t})\right]$, the Orbit Acceleration Parameter $\gamma_{i}(t)$ reduces to,

$$
\gamma_{\mathrm{i}}(\mathrm{t}) \cong\left[2 / \mathrm{M}_{\mathrm{i}}(\mathrm{t})\right] \frac{d}{d t}\left[\frac{d}{d t} \mathrm{M}_{\mathrm{i}}(\mathrm{t})\right]
$$

\section{References}

[1] Hubble Edwin, 'A relation between distance and radial velocity among extra-galactic nebulae', Proc. N. A. S., 1929.

[2] Wikipedia.com, 'Hubble’s Law' Sept. 27, 2014.

[3] Wikipedia.com, 'History of Big-Bang Theory' Oct. 27, 2014.

[4] Wikipedia.com, 'Accelerating Universe' Oct. 27, 2014.

[5] www.aip.org/ history/ cosmology/ideas/ expanding.htm, 'The Expanding Universe', Oct 27, 2014

[6] Barnham Keith, 'The Burning Answers', 2014, Weidenfeld \& Nicolson, London

[7] Hawkin Stephen and Leonard Moldinow, 'The Grand Design', 2010, Bantam Books, New York.

[8] Hartston William, 'Things That Nobody Knows', 2011, Atlantic Books, London. 